\title{
Opportunities and Challenges for Current Developmental Neuroscience
}

Niko Steinbeis ${ }^{1} \&$ Daniel S. Margulies ${ }^{2}$

1 Department of Social Neuroscience; 2 Max Planck Research Group for Neuroanatomy \& Connectivity, Max Planck Institute for Human Cognitive and Brain Sciences, Leipzig, Germany.

\begin{abstract}
While developmental neuroscience can often be motivated by clinical and policy concerns, its constituent research methodologies aim to provide insights into the limits and potential of the developing brain. Our article addresses two main approaches for characterizing psychological and neural changes that occur between infancy and adolescence. Specifically, with respect to psychological change, frequently debated topics such as the nature of developmental continuity can potentially gain from the insights of brain data. Whereas for neuroimaging approaches, which have gained substantial traction in recent years, the advances in describing the developing 'connectome' has been challenged by awareness of imaging artifacts related to behavioral aspects of development (such as motion). As these two fields (developmental psychology and neuroscience) continue to integrate by, for example, constraining psychological hypotheses with brain data and offering explanatory models of neuroimaging findings, the resolution of these challenges charts the development of the field itself.
\end{abstract}

\section{Table of Contents}

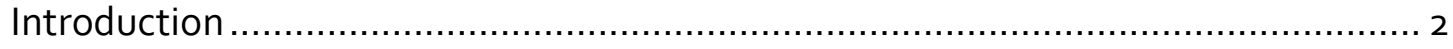

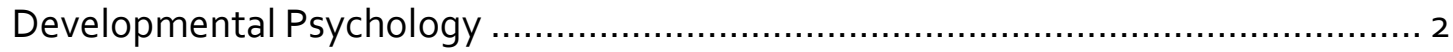

On the nature of change in developmental psychology ................................... 3

Developmental change in theory of mind ................................................. 4

Using cognitive neuroscience for developmental cognitive theory ..................... 5

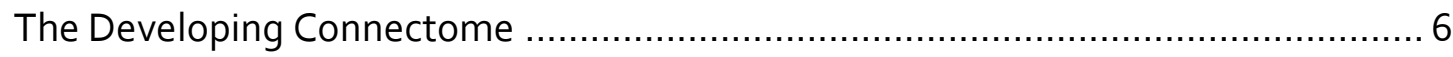

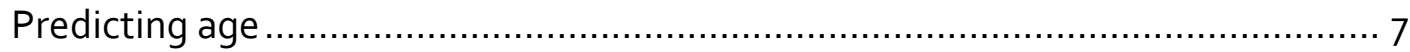

Integrating developmental connectomics and cognitive psychology .................. 8

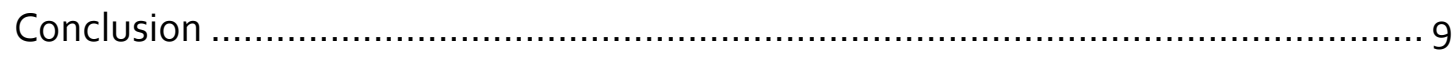

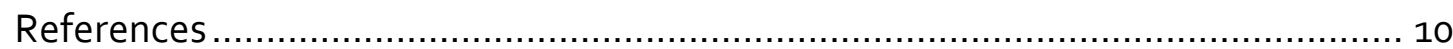




\section{Introduction}

Child development is characterized by significant changes across all domains of psychological functioning. Whereas it is evident that change occurs, how exactly it does so is much less clear and subject to continuous debates (Kagan, 1980). Thus, it remains an open question if the type of developmental process might be unique to a particular period or if it is shared with other later periods. For instance, is the way a young child tries to make sense of its social environment and understands the workings of other minds comparable to the way that an adolescent might perform such a feat? Thus, developmental process can occur through a series of distinct stages, each qualitatively different from one another or as a continuous process. For instances changes in verbal competence or social behavior could result from incremental steps in core abilities. This may differ depending on the phenomenon under investigation. Addressing such a question poses major theoretical and methodological challenges to researchers, given that what is required are longitudinal designs with paradigms that capture the same ability to the same extent at various points in time, but still ultimately rely on a statistical relationship between two measures tapping into the same behavioural surface feature over time.

The study of the developing brain has progressed owing to innovations in non-invasive neuroimaging technologies over the past two decades, enabling questions regarding the relation between child psychological and neural development to be investigated. Alongside the various longitudinal measures of developing brain anatomy and functional activation patterns relating to task performance is a rapidly emerging line of research into the human connectome (Sporns, Tononi, \& Kotter, 2005) - or set of neural connections - and the changes it undergoes throughout development (Power, Fair, Schlaggar, \& Petersen, 2010). Investigation of the developing connectome has pivoted developmental neuroscience from the conceptual framework of psychology and towards the analytic tools of network theory. Complementing psychological debates regarding the potentially divergent mechanisms underlying similar behaviors at various points in development, connectomic research unveils shifts in brain organization throughout childhood (Fair et al., 2009), requiring a novel understanding of how this dynamic functional anatomy is characterized. Just as it would be inaccurate to assume the same psychological mechanisms underlie corresponding faculties, current advances in developmental neuroscience pose a core challenge to the static notion of functional roles.

The following paper takes the shifting terrain of developmental cognitive neuroscience as its starting point. We bring our complementary disciplinary foundations (developmental cognitive psychology and systems neuroscience) into dialogue to interrogate the current challenges facing our respective fields, their trajectories, and possibilities for jointly addressing research questions.

\section{Developmental Psychology}

We would like to begin by arguing that developmental cognitive neuroscience is in a unique position to contribute to psychological theory given that it provides evidence on the stability or variability of core neural mechanisms subserving specific 
psychological functions. Arguably, the debate over the nature of change in development has raged most fiercely in the study of personality development over the lifespan (Caspi, Roberts, \& Shiner, 2005). We will discuss this tenet with reference to another ability that has been at the center of this debate in child development, namely theory of mind (ToM). We will begin by outlining some basic and relevant theoretical concepts of developmental change. This will be followed by the main theoretical controversies regarding developmental changes of ToM. A subsequent summary of neuroscientific findings in the field of ToM will set the stage for making a set of key predictions that would be able to provide evidence in favour of either one of the two positions on the nature of change in child development.

\section{On the nature of change in developmental psychology}

Continuity and discontinuity are two fundamental concepts of understanding and describing change in the course of human development (Emde \& Harmon, 1984). In this context change refers to the emergence of new functions and abilities across cognitive or motor domains, such as the acquisition of object permanence, the ability to recognize one's mirror image or learning to walk. A crucial question in this context is whether the change is fundamental and constitutes the substitution of an already acquired ability with a newly acquired one (i.e. crawling by walking) or rather entails the addition or modification of an already acquired ability (i.e. acquiring the accurate use of syntactic structures; Flavell, 1982; Kagan, 1981; Piaget, 1971). The work of Jean Piaget bears the hallmarks of someone arguing for connectivity of change, where the child's interactions with the environment based on the present state of knowledge will lead to increased abstraction over a larger range of different contexts out of which new insight ultimately emerge as a constituent of a new developmental stage (Piaget, 1952). The influential work of attachment theorist John Bowlby suggests that the attachment styles are highly stable across the life-span (Bowlby, 1969), subject to relatively little change, and thus evidence for stability in important socio-affective functions (Waters, Merrick, Treboux, Crowell, \& Albersheim, 2000). Others however have argued against the parsimony of developmental continuity (Kagan, 1980), making a case for the idea that notions of continuity are more parsimonious with the history of western thought and therefore more amenable as an interpretative heuristic when discussing the nature of change in human development (Kagan, 1980).In support of this, studies on changes in attachment style over the lifespan can be cited, whereby it has been shown that this occurred as a function of negative life events (Waters et al., 2000).

A crucial question in this discussion pertains to the kind of methodology and evidence required to allow making an inference on the nature of change. One key criticism levelled at developmental psychologists is the affinity to seeing relationships based on surface features of the studied behavior (Kagan, 1980). Thus, surface features such as correlations between two measures of the same construct at two time points suggestive of a stable relationship over time may provide only inadequate evidence on whether a skill at time point one is fundamentally the same at time point two, given the influence of unobserved latent variables. In fact, debates on the type of evidence required to decide on the nature of change frequently appear to end up in this impasse. We suggest that cognitive neuroscience 
has much to offer by providing information on the similarity of the underlying neural mechanisms subserving specific cognitive functions. We make this case by discussing the literature on the development of theory of mind.

\section{Developmental change in theory of mind}

Normal human adults are able to attribute mental states to one another. This ability has also been referred to as possessing a theory of mind and has been the subject of intense and unabated scientific scrutiny for the last four decades (Dennett, 1978). Particularly the question how humans come to develop this capacity has played a dominant role in developmental cognitive psychology (Flavell, 1999; Saxe, Carey, \& Kanwisher, 2004; Wimmer \& Perner, 1983). The classic test of this ability in development is known as the false-belief task (Dennett, 1978; Wimmer \& Perner, 1983). In standard versions of this task, the child is told a story of a character whose belief about an object's location is initially correct, but becomes false as a function of the object being moved without the character's knowledge. For example, Sally has a ball and decides to put the ball into a basket. While Sally leaves the scene Anne decides to move the ball from the basket to a nearby box. Upon Sally's return the child is asked where Sally will look for the ball. To accurately respond, the child, who knows where the ball is, has to track the beliefs of the character in question and simultaneously inhibit its own knowledge of where the desired object is hidden. Variants of this task abound and the findings converge that until around the age of four years children do not respond correctly to the question. As a result, it has been prominently argued, that between the age of 3-4 years children undergo a fundamental change in the concepts used to reason about and understand other minds and that such a now representational concept was absent until then (Flavell, 1999; Perner, 1993; Saxe et al., 2004; Wellman, Cross, \& Watson, 2001). Whereas credence is typically given to the development of potential precursors of a fullfledged ToM, such as understanding other's perceptions, emotions, desires and goals, these are argued to be functionally distinct (Saxe et al., 2004). The basic argument here is that the unique accomplishment of attributing mental states to others occurs as a function of a fundamental organizational change in the underlying cognitive architecture, giving rise to the child's ability to posit a theory of other's mental states (Gopnik \& Meltzoff, 1997).

Such accounts have been questioned especially, because the high cognitive demands on executive functions and language comprehension may have masked the possession of theory of mind in younger children (Bloom \& German, 2000). Thus, poor performance of younger children may not reflect the fundamental absence of a particular social ability per se, but result from inadequate skills required to perform on such tasks. In line with such proposals, recent studies have garnered evidence by means of so-called violation of expectation paradigms, which show through recording looking times that infants as young as 15 months seem to have an understanding of others' false-beliefs (Onishi \& Baillargeon, 2005). More recent and methodologically less contentious anticipatory looking paradigms have been able to buttress claims of early false-belief understanding in young infants (Southgate, Senju, \& Csibra, 2007). By now there is a substantial amount of 
evidence suggesting that children younger than four years are able to track others' beliefs, culminating in the observation that seven-month old infants are already influenced by the beliefs of other agents in responding to the presence or absence of visual stimuli (Kovacs, Teglas, \& Endress, 2010). Some would argue that there is no fundamental change around four years of age and that earlier versions provide the "conceptual foundation" for later abilities to reason about false-beliefs (e.g. early representational abilities in infants are built upon leading to a capacity for metarepresentation, forming the basis for a theory of mind; Leslie, 1987; Surian, Caldi, \& Sperber, 2007) Others however question whether infants as young as 15 or even 7 months can already be said to be tracking other's beliefs (Perner \& Ruffman, 2005), suggesting that instead infants assign a set of behavioural rules, which do not necessarily comprise a mediating mental state. To provide a reconciliation to these positions, it has been proposed recently that there might actually be two cognitive systems in place, one for tracking full-fledged beliefs and another tracking belieflike states (Apperly \& Butterfill, 2009; Rakoczy, 2012). Thus, the relationship between early and late competencies of ToM and if the two are the same, only partly so or fundamentally distinct continues to be controversial, while the type of evidence required to allow to argue for either remains opaque. Even though longitudinal data suggest that ontogenetically early and late false-belief competencies are linked (Thoermer, Sodian, Vuori, Perst, \& Kristen, 2012) covarying changes in unobserved latent variables render this type of evidence as less than suitable to settle the debate.

\section{Using cognitive neuroscience for developmental cognitive theory}

The utility of cognitive neuroscience to reliably infer cognitive processes from the neuronal function of specific brain regions has made enormous progress. The fact that particular brain regions are functionally dedicated to perform specific cognitive computations is one of the basic assumptions of the present theoretical claim. This is substantiated byrecent developments in the analyses of large-scale data sets and across meta-analyses of studies, which allow for the reliable and consistent mapping of cognitive process with specific brain regions (Yarkoni, Poldrack, Nichols, Van Essen, \& Wager, 2011). Thus, using both forward- and reverse inference it is possible to predict which brain regions are differentially recruited not just for lower level sensory processes such as pain processing, but also for higher-level cognitive and affective processes, such as working memory (Yarkoni et al., 2011). In the case of theory of mind there is by now an abundance of studies implicating a circumscribed network of brain regions required for tracking and computing others' beliefs in adults. These include the medial prefrontal cortex (mPFC), the bilateral temporo-parietal junction (TPJ), the superior temporal sulcus (STS) as well as the temporal pole (TP; Frith \& Frith, 2003). Recent attempts have been made to decompose this network into the subcomponential processes required for belief attribution (i.e. representing other's belief, decoupling own belief from that of others, inhibiting own belief; Frith \& Frith, 2003). Thus, some have argued for a unique role of TPJ in representing others' beliefs (Saxe \& Kanwisher, 2003), while this has been contended by others (Mitchell, 2008). Regardless of the precise role of specific brain regions in the context of mental state attribution, there is by now solid evidence in support of a network of regions reliably activated when making 
inferences of others mental states .(Bzdok et al., 2012; Schurz, Radua, Aichhorn, Richlan, \& Perner, 2014).

Such a persistent association of a circumscribed set of brain regions with a specific cognitive function allows for making predictions over the course of development, because accounts of developmental continuity and discontinuity would make different predictions with which brain areas ought to be observed over time. Developmental continuity of theory of mind would predict that core processes are the same over development, whereas developmental discontinuity states that these differ and change so fundamentally as to be represented in a qualitatively different manner. Thus, if a function develops continuously the same brain regions should be implicated at the various time points, whereas if development occurs discontinuously, this should manifest itself in a different set of brain regions. Recent findings from resting-state analyses in both adults and children exemplify this point perfectly by showing that neural networks typically associated with TOM are present to a comparable extent in children aged 6 to 12 years and adults (Steinbeis, Bernhardt, \& Singer, in press). These findings are complemented by behavioral findings of a continuity of TOM development from child- to adulthood starting at 6 years of age in that adults and older children were shown to find it as difficult as younger children to process an agent's false belief compared to a true belief (Apperly, Warren, Andrews, Grant, \& Todd, 2011). Relating this back to our initial example of ToM development, if like many developmental cognitive psychologists believe that a full-fledged theory of mind emerges uniquely around the age of four years and is fundamentally different to any such ability shown at younger ages, different brain regions should be associated with the two abilities. If however, the change from early abilities of attributing belief-like states were a functional antecedent to the fully developed theory of mind, we would expect to see this become evident in the recruitment of the same brain regions.

There are of course some caveats that are important to bear in mind with such an approach. For instance, the possibility of age-related changes in the cortical representation of the same cognitive function may lead to different brain regions subserving the same process over time. This constraint implies that cognitive neuroscience would only be able to inform on whether the neurocognitive development of theory of mind abilities is continuous (i.e. the same brain region subserves theory of mind performance at both time points). Nonetheless, our reasoning suggests, that cognitive neuroscience is in a unique position to offer the kind of evidence required to settle the debate on whether early competencies in theory of mind are linked not just at a surface level but at a deep structural level indicative of shared cognitive processes.

\section{The Developing Connectome}

Task-related activation provides one means for assessing the trajectory of alterations in brain function. The connectomic approach offers another means based on the relationship between connectivity and function. In order to study developmental changes in brain function, the premise of this line of research is that functional changes can be inferred from unique patterns of connectivity. 
Rather than describe the merits and costs of this approach, we offer an overview of the novel methods and findings related to characterizing changes during development using maps of connectivity. A method termed resting-state fMRI, in particular, has gained prominence for its ease of acquisition and flexibility in post hoc analyses. Based in the correlation of spontaneous intrinsic brain activity, resting-state $\mathrm{fMRI}$ offers a means to describe the functional connectivity throughout the brain using as little as five minutes of data acquisition time. When considering the particular challenges of the MRI scanning environment - especially the requirement to remain absolutely still throughout the duration of the scan - the short scan time provides a significant advantage in studying children.

The literature addressing connectome development in childhood ranges in scope from addressing alterations in specific cognitively-defined networks, to attempts to generalize principles of brain organization using techniques from graph theory. For example, in a series of publications by Damian Fair and colleagues, the research agenda shifts from being strongly rooted in cognitive developmental models, and investigating their corresponding control (Fair et al., 2007) and default-mode networks (Fair et al., 2008), to more network theory-based abstractions that describe development as a local-to-distributed developmental shift in network organization (Fair et al., 2009). In readdressing our question of developmental trajectories, here with respect to network models of brain organization, it has the potential to inform psychological questions for understanding the time course of network alterations.

\section{Predicting age}

Similar conceptual shifts that allow the network, rather than the cognitive models themselves, to constitute the basis of developmental cognitive neuroscience have been further underlined by aims to predict age, rather than to describe it, using brain connectivity. While previous attempts to predict diagnostic categories from functional connectivity data - most notably with the outcome of the ADHD-200 competition to predict attention-deficit/hyperactivity disorder subtypes demonstrated far less then optimal performance of current machine learning algorithms, the prediction of chronological age has proven more effective. The watershed study to describe the prediction of age using functional connectivity data coined this normative arc as the "functional brain maturation curve" (Dosenbach et al., 2010). While not the first study to describe various linear and higher order relationships between network organization and age (e.g., Kelly et al., 2009; Zuo et al., 2010), framing the metric as predictive transferred the status from experimental to clinically relevant.

Methodological controversy has arisen regarding the validity of these findings for describing actual brain connectivity in developmental studies (Power, Barnes, Snyder, Schlaggar, \& Petersen, 2012). The core issue relates to the effect of motion on functional connectivity measures, which can artifactually decrease the strength of long-distance connections and increase those of short-distances. While numerous studies over the past two years have debated the true motion-related impact on various group-difference and developmental studies, the correlation between childhood age and the ability to remain still in the scanner environment is 
without question. As long-distance connections form the network nodes of the networks largely implicated in developmental studies, the motion effects on longdistance anterior-posterior cortical networks are particularly challenging to current findings.

\section{Integrating developmental connectomics and cognitive psychology}

One prominent theory of the developmental relationships between connectomic and functional organization is the theory of neural constructivism, which argues that cognitive improvements result from greater connectivity between increasingly functionally specialized areas during development (Stevens, 2009). While this offers an explanation for lower-level sensory specialization, the developmental changes in cognitive faculties, such as ToM, would need to recruit additional systems in a context dependent and flexible manner. As neural constructivism does not account for this prolonged possibility for system-wide flexibility, it would be necessary to integrate theories of flexible and context-dependent functional integration to the neural constructivist model. The functional connectivity methods described above would be optimal for describing the neural substrates of cognitive development by offering information for how and why network alterations promote optimal cognitive abilities throughout development.

In addressing the development of ToM, though no focused study yet exists, several functional connectivity findings provide insight into related network alterations. Medial prefrontal cortex is implicated in functions related to social cognition, and its investigation provides one means of assessing connectivity changes related to an aspect of ToM. Kelly et al. (2009) found that a related area of the perigenual cingulate demonstrates its long-distance connections latest in development from childhood to early adulthood when compared to other areas of the anterior cingulate. Furthermore, the specific connectivity that develops is consistent with the areas of the ToM-related network.

Of course, given that mere behavioural observations to resolve questions on developmental continuity were critiqued on methodological grounds, we must be equally rigorous with the present approach. First, developmental imaging is a challenge no matter what the age group or phenomenon under investigation. However, this challenge increases considerably the younger the participants, but even for children as young as 24 months, this is not impossible. Whereas acquiring task-related functional activation for children that young is unrealistic, task performance can be related to task-free resting state functional magnetic resonance imaging ( $\mathrm{rs}-\mathrm{fMRI}$ ) connectivity as well as structural imaging. Thus parameters such as degree centrality and functional connectivity in rs-fMRI and cortical thickness and structural covariance as well as fibre anisotropy and diffusivity provide excellent measures of the functional relevance of specific brain regions in the performance of a particular task. Degree centrality of a specific brain region for instance can provide information on the importance of a brain region within a hierarchical cluster of connected brain regions. Thus, one pattern of continuous development of theory of mind associated brain regions could be a correlation in degree centrality of MPFC or TPJ with performance on non-verbal tasks of ToM abilities, which would persist at a later time point with performance on verbal and 
explicit tasks of ToM abilities. Importantly, concepts of continuous development of addition and modification would not preclude slight age-related changes in the topology of the functional network within which brain regions that are both associated with ToM abilities and possess high functional degree centrality are embedded. As long as certain core features are retained, this should be taken as an indicator of developmental continuity.

\section{Conclusion}

It is often asked how exactly brain imaging methodologies can inform psychological theory, given the considerable costs associated with measurement, data storage and processing. We have tried to outline one specific case where cognitive neuroscience can be informative over and above behavioural paradigms on topics that are central to theories of cognitive development. Thus, developmental neuroscience can carve out a unique position in being able to inform on issues related to the mechanisms and nature of change. 


\section{$\underline{\text { References }}$}

Apperly, I. A., \& Butterfill, S. A. (2009). Do Humans Have Two Systems to Track Beliefs and Belief-Like States? Psychological Review, 116(4), 953-970. doi: Doi 10.1037/A0016923

Apperly, I. A., Warren, F., Andrews, B. J., Grant, J., \& Todd, S. (2011).

Developmental Continuity in Theory of Mind: Speed and Accuracy of BeliefDesire Reasoning in Children and Adults. Child Development, 82(5), 1691-1703.

Bloom, P., \& German, T. P. (2000). Two reasons to abandon the false belief task as a test of theory of mind. Cognition, 77(1), B25-B31. doi: Doi 10.1016/Soo100277(00)00096-2

Bowlby, J. (1969). Attachment. New York: Basic Books.

Bzdok, D., Schilbach, L., Vogeley, K., Schneider, K., Laird, A. R., Langner, R., \& Eickhoff, S. B. (2012). Parsing the neural correlates of moral cognition: ALE meta-analysis on morality, theory of mind, and empathy. Brain Structure \& Function, 217(4), 783-796. doi: 10.1007/500429-012-0380-y

Caspi, A., Roberts, B. W., \& Shiner, R. L. (2005). Personality development: Stability and change. Annual Review of Psychology, 56, 453-484. doi: DOI 10.1146/annurev.psych.55.090902.141913

Dennett, D. C. (1978). Cognition and Consciousness in Nonhuman Species Comment. Behavioral and Brain Sciences, 1(4), 568-570.

Dosenbach, N. U., Nardos, B., Cohen, A. L., Fair, D. A., Power, J. D., Church, J. A., . . . Schlaggar, B. L. (2010). Prediction of individual brain maturity using fMRI. Science, 329(5997), 1358-1361. doi: 10.1126/science.1194144

Emde, R. N., \& Harmon, R. J. (1984). Continuities and Discontinuities in Development. New York: Plenum Press.

Fair, D. A., Cohen, A. L., Dosenbach, N. U., Church, J. A., Miezin, F. M., Barch, D. M., ... Schlaggar, B. L. (2008). The maturing architecture of the brain's default network. Proc Natl Acad Sci U S A, 105(10), 4028-4032. doi: 10.1073/pnas.0800376105

Fair, D. A., Cohen, A. L., Power, J. D., Dosenbach, N. U., Church, J. A., Miezin, F. M., ... Petersen, S. E. (2009). Functional brain networks develop from a "local to distributed" organization. PLoS Comput Biol, 5(5), e1000381. doi: 10.1371/journal.pcbi.1000381

Fair, D. A., Schlaggar, B. L., Cohen, A. L., Miezin, F. M., Dosenbach, N. U., Wenger, K. K., ... Petersen, S. E. (2007). A method for using blocked and event-related fMRI data to study "resting state" functional connectivity. Neuroimage, 35(1), 396-405. doi: 10.1016/j.neuroimage.2006.11.051 
Flavell, J. H. (1982). Structures, stages, and sequences in cognitive development. In W. A. Collins (Ed.), The concept of development. Hillsdale, N.J.: Lawrence Erlbaum.

Flavell, J. H. (1999). Cognitive development: Children's knowledge about the mind. Annual Review of Psychology, 50, 21-45. doi: DOI 10.1146/annurev.psych.50.1.21

Frith, U., \& Frith, C. D. (2003). Development and neurophysiology of mentalizing. Philosophical Transactions of the Royal Society B-Biological Sciences, 358(1431), 459-473. doi: DOI 10.1098/rstb.2002.1218

Gopnik, A., \& Meltzoff, A. (1997). Words, thoughts and theories. Cambridge, MA: MIT Press.

Kagan, J. (1980). Perspectives on Continuity. In O. G. Brim \& J. Kagan (Eds.), Constancy and Change in Human Development. Cambridge, Mass: Harvard University Press.

Kagan, J. (1981). The second year: The emergence of self-awareness. Cambridge, Mass: Harvard University Press.

Kelly, A. M. C., Di Martino, A., Uddin, L. Q., Shehzad, Z., Gee, D. G., Reiss, P. T., .. . Milham, M. P. (2009). Development of anterior cingulate functional connectivity from late childhood to early adulthood. Cereb Cortex, 19(3), 640-657. doi: 10.1093/cercor/bhn117

Kovacs, A. M., Teglas, E., \& Endress, A. D. (2010). The Social Sense: Susceptibility to Others' Beliefs in Human Infants and Adults. Science, 330(6012), 1830-1834. doi: DOI 10.1126/science.1190792

Leslie, A. M. (1987). Pretense and Representation - the Origins of Theory of Mind. Psychological Review, 94(4), 412-426. doi: Doi 10.1037/0033-295X.94.4.412

Mitchell, J. P. (2008). Activity in right temporo-parietal junction is not selective for theory-of-mind. Cerebral Cortex, 18(2), 262-271. doi: 10.1093/cercor/bhmo51

Onishi, K. H., \& Baillargeon, R. (2005). Do 15-month-old infants understand false beliefs? Science, 308(5719), 255-258. doi: DOI 10.1126/science.1107621

Perner, J. (1993). Understanding the Representational Mind. Cambridge, Massachusetts: MIT Press.

Perner, J., \& Ruffman, T. (2005). Infants' insight into the mind: How deep? Science, 308(5719), 214-216. doi: DOI 10.1126/science.1111656

Piaget, J. (1952). The origins of intelligence. New York: International Universities Press.

Piaget, J. (1971). Biology and Knowledge. Chicago: University of Chicago Press. 
Power, J. D., Barnes, K. A., Snyder, A. Z., Schlaggar, B. L., \& Petersen, S. E. (2012). Spurious but systematic correlations in functional connectivity MRI networks arise from subject motion. Neuroimage, 59(3), 2142-2154. doi:

10.1016/j.neuroimage.2011.10.018

Power, J. D., Fair, D. A., Schlaggar, B. L., \& Petersen, S. E. (2010). The development of human functional brain networks. Neuron, 67(5), 735-748. doi: 10.1016/j.neuron.2010.08.017

Rakoczy, H. (2012). Do infants have a theory of mind? British Journal of Developmental Psychology, 30(1), 59-74. doi: DOI 10.1111/j.2044835X.2011.02061.X

Saxe, R., Carey, S., \& Kanwisher, N. (2004). Understanding other minds: Linking developmental psychology and functional neuroimaging. Annual Review of Psychology, 55, 87-124. doi: DOI 10.1146/annurev.psych.55.090902.142044

Saxe, R., \& Kanwisher, N. (2003). People thinking about thinking people - The role of the temporo-parietal junction in "theory of mind". Neuroimage, 19(4), 18351842. doi: Doi 10.1016/S1053-8119(03)00230-1

Schurz, M., Radua, J., Aichhorn, M., Richlan, F., \& Perner, J. (2014). Fractionating theory of mind: a meta-analysis of functional brain imaging studies. Neurosci Biobehav Rev, 42, 9-34. doi: 10.1016/j.neubiorev.2014.01.009

Southgate, V., Senju, A., \& Csibra, G. (2007). Action anticipation through attribution of false belief by 2-year-olds. Psychol Sci, 18(7), 587-592. doi: 10.1111/j.1467$9280.2007 .01944 \cdot x$

Sporns, O., Tononi, G., \& Kotter, R. (2005). The human connectome: A structural description of the human brain. PLoS Comput Biol, 1(4), e42. doi: 10.1371/journal.pcbi.0010042

Steinbeis, N., Bernhardt, B. C., \& Singer, T. (in press). Structural Immaturity of rSMG and Reduced Functional Connectivity with DLPFC explains Heightened Affective Egocentricity Bias in Childhood. Social Cognitive and Affective Neuroscience.

Stevens, M. C. (2009). The developmental cognitive neuroscience of functional connectivity. Brain Cogn, 70(1), 1-12. doi: 10.1016/j.bandc.2008.12.009

Surian, L., Caldi, S., \& Sperber, D. (2007). Attribution of beliefs by 13-month-old infants. Psychol Sci, 18(7), 580-586. doi: DOI 10.1111/j.1467-9280.2007.01943.x

Thoermer, C., Sodian, B., Vuori, M., Perst, H., \& Kristen, S. (2012). Continuity from an implicit to an explicit understanding of false belief from infancy to preschool age. British Journal of Developmental Psychology, 30(1), 172-187. doi: DOI 10.1111/j.2044-835X.2011.02067.X 
Waters, E., Merrick, S., Treboux, D., Crowell, J., \& Albersheim, L. (2000).

Attachment security in infancy and early adulthood: A twenty-year longitudinal study. Child Development, 71(3), 684-689. doi: Doi 10.1111/1467-8624-00176

Wellman, H. M., Cross, D., \& Watson, J. (2001). Meta-analysis of theory-of-mind development: The truth about false belief. Child Development, 72(3), 655-684. doi: Doi 10.1111/1467-8624.00304

Wimmer, H., \& Perner, J. (1983). Beliefs About Beliefs - Representation and Constraining Function of Wrong Beliefs in Young Childrens Understanding of Deception. Cognition, 13(1), 103-128. doi: Doi 10.1016/0010-0277(83)90004-5

Yarkoni, T., Poldrack, R. A., Nichols, T. E., Van Essen, D. C., \& Wager, T. D. (2011). Large-scale automated synthesis of human functional neuroimaging data. Nature Methods, 8(8), 665-U695. doi: Doi 10.1038/Nmeth.1635

Zuo, X. N., Kelly, C., Di Martino, A., Mennes, M., Margulies, D. S., Bangaru, S., ... Milham, M. P. (2010). Growing together and growing apart: regional and sex differences in the lifespan developmental trajectories of functional homotopy. $J$ Neurosci, 30(45), 15034-15043. doi: 10.1523/JNEUROSCI.2612-10.2010 